\title{
BMJ Open Effects of physical activity and sedentary behaviour on cardiometabolic risk factors and cognitive function in children: protocol for a cohort study
}

\author{
Yajie Lv (D),${ }^{1}$ Li Cai, ${ }^{1}$ Zhaohuan Gui, ${ }^{1}$ Xia Zeng, ${ }^{1}$ Minyi Tan, ${ }^{1}$ Nianqing Wan, ${ }^{1}$ \\ Lijuan Lai, ${ }^{1}$ Shaomin Lu, ${ }^{2}$ Weiqing Tan, ${ }^{3}$ Yajun Chen ${ }^{1}$
}

To cite: Lv Y, Cai L, Gui Z, et al. Effects of physical activity and sedentary behaviour on cardiometabolic risk factors and cognitive function in children: protocol for a cohort study. BMJ Open 2019;9:e030322. doi:10.1136/ bmjopen-2019-030322

- Prepublication history for this paper is available online. To view these files, please visit the journal online (http://dx.doi. org/10.1136/bmjopen-2019030322).

Received 11 March 2019 Revised 28 June 2019 Accepted 07 August 2019

D Check for updates

(C) Author(s) (or their employer(s)) 2019. Re-use permitted under CC BY-NC. No commercial re-use. See rights and permissions. Published by BMJ.

${ }^{1}$ Department of Maternal and Child Health, School of Public Health, Sun Yat-sen University, Guangzhou, China

${ }^{2}$ Department of Child Health, Guangzhou Women and Children's Medical Center, Guangzhou, China

${ }^{3}$ Health Promotion Center for

Primary and Secondary Schools of Guangzhou Municipality, Guangzhou, China

Correspondence to

Dr Yajun Chen;

chenyj68@mail.sysu.edu.cn

\section{ABSTRACT}

Introduction Although studies showed that physical activity (PA) and sedentary behaviour (SB) were associated with cardiometabolic risk factors and cognitive function, both independent and combined associations among them are inconsistent. Cardiometabolic risk factors are also associated with cognitive function, but research of children is limited. Additionally, the brain level mechanisms have not been fully established. The proposed study aims to explore the associations and mechanisms of PA and SB on cognitive function and cardiometabolic risk factors in children.

Methods and analysis This is a school-based prospective cohort study. A total of 8324 participants of this study are primary school students aged 7-12 years old who are followed up every 2 years from January 2017 to December 2026. We used a stratified cluster random sampling to select five primary schools in Guangzhou, China. There are three phases at baseline. At phase I, we collect PA, SB and cognitive function by questionnaires and also conduct anthropometric and biochemical measurements in all participants. At phase II, PA, SB and cognitive function are measured respectively by accelerometers and cognitive tasks among participants randomly selected from four subgroups with different SB and PA levels. At phase III, event-related potentials are recorded using electroencephalogram during a cognitive task among participants randomly selected from phase II. We plan to follow-up all participants until they graduate from high school. The process applied at baseline and follow-up are approximately identical.

Ethics and dissemination Procedures described in this manuscript have been approved by the Ethical Review Committee for Biomedical Research, School of Public Health, Sun Yat-sen University (L2016-010). All parents or guardians of participants signed the informed consent form voluntarily before participating in the study. The findings of the study will be published in peer-reviewed journals.

Trial registration number NCT03582709

\section{INTRODUCTION}

Physical activity (PA) levels were declining and sedentary behaviour (SB) levels were rising among children in China in recent

\section{Strengths and limitations of this study}

- This is a prospective longitudinal study providing greater power to determine the effects of physical activity (PA) and sedentary behaviour (SB) on cardiometabolic risk factors and cognitive function over different development time points in children.

- We use a combination of subjective and objective measurements to provide quantitative and qualitative details from multiple dimensions.

- Samples were stratified into four subgroups with different PA and SB levels so that the independent and combined associations of SB and PA can be studied.

- It is not a representative sample because the registration is conducted among the schools in the specific area.

decades. ${ }^{1}$ More than $80 \%$ of adolescents across the globe could not achieve the WHO's PA recommendation of $60 \mathrm{~min} /$ day. $^{2}$ About $65 \%$ children spend 2 hours/day or more on watching television, while over a third of children spend 3 hours/day or more on $\mathrm{SB} .{ }^{3}$ Higher levels of moderate-to-vigorous physical activity (MVPA) can reduce the risk of cardiometabolic factors, regardless of the amount of time spent SB. ${ }^{45}$ However, SB is also suggested as an emerging and unique determinant of cardiometabolic diseases in children, independent of MVPA. ${ }^{6}$ Further studies are warranted to determine the independent and combined associations between $\mathrm{PA}$ and $\mathrm{SB}$ with cardiometabolic risk factors in children.

There is a growing number of studies investigating the associations of $\mathrm{PA}$ and $\mathrm{SB}$ on psychological consequences during development, especially on cognitive function in children. Meta-analyses showed a significant positive relationship between PA and cognitive function in children, such as executive 
functions, attention and academic performance. ${ }^{78}$ Several physiological mechanisms including changes in vascularity ${ }^{9}$ and psychosocial mechanisms could explain the positive effects of PA on cognitive function. Conversely, previous study has shown a detrimental association between SB and cognitive function, ${ }^{10}$ but further studies suggested that different volume, pattern and forms of SB may have different impacts on cognitive development. ${ }^{11} 12$ The associations of SB and cognitive function are complex and the brain levels mechanisms also have not been fully established. Neurophysiological studies have revealed that physical activity influenced baseline electrocortical function and, thus, examination of electroencephalograms (EEGs) might provide evidence on brain level of cognitive operations. ${ }^{13}$

Cardiometabolic risk factors, including obesity, ${ }^{14}$ dyslipidaemia, ${ }^{15}$ altered glucose metabolism ${ }^{16}$ and hypertension ${ }^{17}$ were also associated with impaired cognitive function. The detrimental associations have been most fully demonstrated among adults. ${ }^{18} 19$ Meanwhile, the mechanism study showed that PA changes the vascularity and leads to increased oxygen saturation and glucose delivery, promotion of angiogenesis and improved cerebral blood flow to areas related to cognition, alertness and focus. ${ }^{9}$ We can speculate that cardiometabolic risk factors, influenced by SB or PA levels, or both, could have effects on cognitive function in children.

Given this partially unclear situation, the proposed study aims: (1) to evaluate the current situation of PA, SB, cardiometabolic risk factors and cognitive function with primary school students; (2) to clarify the independent and combined association of PA and SB with cardiometabolic risk factors and cognitive function and (3) to explore the brain levels mechanisms of PA and SB on cognitive function by EEG recordings.

\section{Design}

This study was designed as a school-based prospective cohort study. Primary school children aged 7-12 years were recruited and are followed up every 2 years from January 2017 to December 2026. There are three phases at baseline (figure 1).

\section{Research strategies}

Phase I: All students in school are investigated under an epidemiological survey from January 2017 to June 2017. Each student has his or her own identification to match their variable information. Questionnaire information (including an informed consent), biochemical outcomes and anthropometric data are collected. The questionnaire is distributed to children at school, and children and their parents fill it out together at home. Blood samples collection and anthropometric measures are conducted at school. Biochemical analysis is conducted at the laboratory of School of Public Health, Sun Yat-sen University or Health Promotion Center for Primary and Secondary Schools of Guangzhou Municipality. The current situation and the correlation among PA, SB, cardiometabolic risk factors and cognitive function will be analysed.

Phase II: To explore the further performance characteristics of children's cardiometabolic risk factors and cognitive function in different PA and SB levels, we classified participants into four subgroups (high $\mathrm{PA} /$ high $\mathrm{SB}$, high $\mathrm{PA} /$ low $\mathrm{SB}$, low $\mathrm{PA} /$ high $\mathrm{SB}$, low $\mathrm{PA} /$ low $\mathrm{SB}$ ). The detailed dimensions of $\mathrm{PA}$ and $\mathrm{SB}$ are measured more accurately by accelerometer combined with an activity $\log$ (PA-log), and cognitive function is measured more objectively through three paradigms. Cardiorespiratory fitness (CRF) and intelligence test are measured as covariates among the four subgroups. The assessments
Baseline

Epidemiological survey at five primary schools in grade 1 to 6

\begin{tabular}{|c|}
\hline $\begin{array}{c}\text { Phase I } \\
(\mathrm{n}=8324)\end{array}$ \\
\hline $\begin{array}{c}\text { All eligible students at } \\
\text { five school }\end{array}$ \\
\hline $2017.01-2017.06$ \\
\hline $\begin{array}{l}\text { (1) } \begin{array}{l}\text { Questionnaire } \\
\text { survey } \\
\text { (2) Biochemical } \\
\text { measures }\end{array} \\
\text { (3) Anthropomctric } \\
\text { measures }\end{array}$ \\
\hline
\end{tabular}

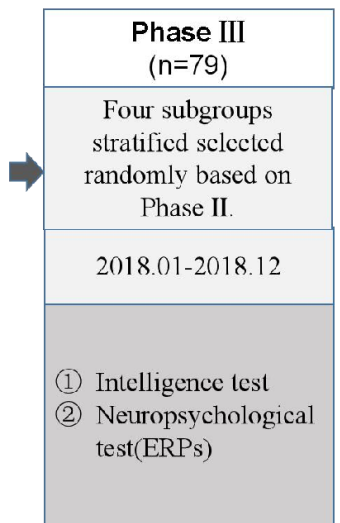

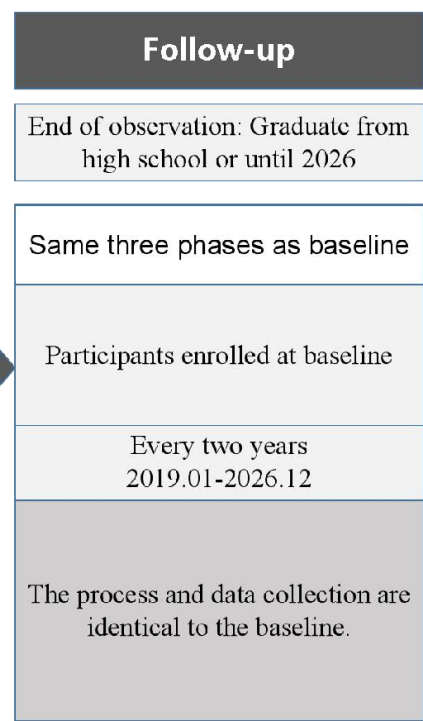

Figure 1 Design of the study.

High SB: SB $\geq$ P75, low SB: SB $<$ P25, high PA: MVPA $\geq 60$ min/day, low PA: MVPA $<150$ min/week. CRF, cardiorespiratory fitness; ERP, event-related potential; MVPA, moderate-to-vigorous physical activity; PA, physical activity; SB, sedentary behaviour. 
are conducted during break time or PA classes at school from July 2017 to December 2017.

Phase III: To explore the underlying neuropsychological mechanisms how do PA and SB impact cognitive function, four subgroups, selected randomly based on phase II are submitted to neuropsychological and electrophysiological assessments. EEG will be used to record event-related potentials (ERPs) for assessing brain level between four subgroups responses to cognitive tasks. Assessments are conducted at the laboratory of School of Public Health, Sun Yat-sen University from January 2018 to December 2018.

\section{Sampling}

Based on our previous studies, it is assumed that the rate of physical inactivity in children was about $49.2 \%$ in Guangzhou. The sample size of the cluster sampling should be increased by half, and the expected invalid response rate is $40 \%$. In terms of stratification according to PA and SB, the calculations showed that a minimum of 3335 people were required. To guarantee that the heterogeneity of social background of the population was represented, a representation of the different school forms in Guangzhou was considered. Hence, the random sequence of schools was computer generated and stratified by school district and school size in each centre. A stratified cluster random sampling was used to select three central urban districts (Yuexiu District, Liwan District, Tianhe District) and two peripheral urban districts (Huangpu District, Panyu District), then respectively chose one ordinary primary school at random from the five districts. At last, we enrolled 8324 students to this cohort study, and the sample size met the requirements.

Participants were categorised into four groups using multiphase stratified probability sampling (figure 2). At first, SB $\geq$ gender-specific, age-specific $75 \%$ percentile and <gender-specific, age-specific $25 \%$ percentile were assigned to the high and low SB subgroup, respectively. Second, based on the WHO standard, participants with MVPA $\geq 60 \mathrm{~min} /$ day were classified into high PA subgroup while those with MVPA $<150 \mathrm{~min} /$ week were classified into low PA subgroup. At last, about 640 children (160 students in each subgroup) were enrolled into the phase II. About 80 children (20 students in each subgroup adjusted for IQ age, gender, body mass index (BMI), CRF) from phase II were enrolled into the phase III by stratified random sampling.

\section{Inclusion and exclusion criteria}

Phase I: Students are excluded if they had: (1) severe physical disease which may have adverse effects on daily physical activity; (2) a mental disease or medical condition leading to unavailability for the questionnaire and (3) severe medical condition that made them absent from school.

Phase II: Inclusion criteria: (1) 7-12 years old; (2) normal visual acuity; (3) right handed. Exclusion criteria: suspected to have developmental behaviour disorders according to the Strengths and Difficulties Questionnaire (SDQ).

Phase III: Children are considered if their IQ $\geq 80$ identified by Wechsler Intelligence Scale for ChildrenFourth Edition (WISC-IV). Other inclusion and exclusion criteria are consistent with the previous phases.

The characteristics of participants at baseline are presented in table 1 .

\section{MEASUREMENTS \\ Phase I \\ Multicomponents questionnaire survey}

The questionnaire was validated in an earlier pilot study that showed acceptable reliability and validity (data not 
Table 1 Baseline characteristics

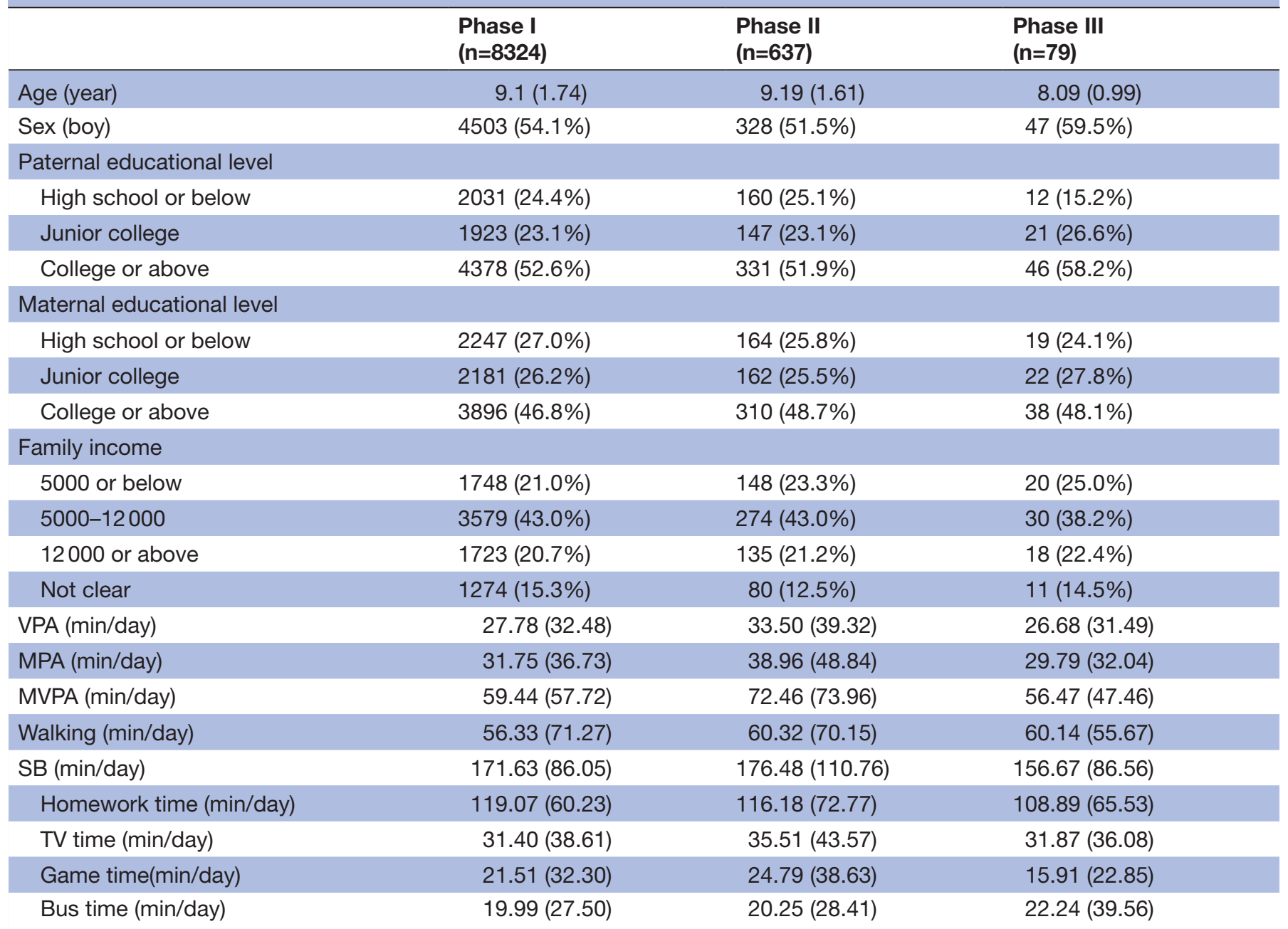

Data are $\mathrm{n}(\%)$ or mean (SD).

MVPA, moderate-to-vigorous physical activity; PA, physical activity; SB, sedentary behaviour.

reported). The questionnaire is consisted of following three parts:

1. Questionnaire on the Health Monitoring of Primary School Students: A self-designed questionnaire is filled out by the children together with their parents for information on sociodemographic, early-life factors, family history, PA, SB, sleeping habits, dietary behaviour, mental health and other possible moderating variables of children. In this part, International Physical Activity Questionnaire Short Form is used to assess the frequency and duration of PA and SB.

2. The SDQ Chinese Version ${ }^{20}$ : To eliminate the children who is suspected to have developmental behaviour disorders, parents are asked to fill out this part.

3. Behaviour Rating Inventory of Executive Function Parent Form: It is an assessment of behaviour at home for children and adolescents aged 5-18 years, and the Chinese version was revised with good reliability and validity by Qian Ying in 2006.
Biochemical measurements

After 12 hours of overnight fast, fasting venous blood samples are taken and collected into EDTA vacuum tubes. Samples are separated within 2 hours of collection centrifuged at $3000 \mathrm{r}$ for $15 \mathrm{~min}$, and plasma or serum are stored immediately at $-80^{\circ} \mathrm{C}$ in the laboratory for future analyses. Five outcomes are measured: fasting glucose, triglyceride, total cholesterol, high-density lipoprotein and low-density lipoprotein. Fasting glucose is measured using the glucose oxidase method, and others are measured using radioimmunoassay kits at the departmental laboratory (HITACHI 7180 automated biochemistry analyser, Tokyo, Japan) at Health Promotion Center for Primary and Secondary Schools of Guangzhou Municipality. All samples are assayed in the same laboratory with the same analysers.

\section{Anthropometric measurements}

Anthropometric variables are measured by professional nurses and doctors according to standard procedures. 
Height and weight are measured wearing light clothes and no shoes. Height is measured by a fixed stadiometer to the nearest millimetre. Weight is measured by a calibrated weighing scale to the nearest kilogram. BMI is calculated as weight $(\mathrm{kg}) /$ height $(\mathrm{m}) .{ }^{2}$ BMI z-score is calculated based on WHO growth reference (2007) ${ }^{21}$ Waist circumference is measured directly on the body surface midway between the lower rib margin and the iliac crest. Additionally, the participants underwent measurement of body composition (Bioelectrical Impedance Analysis by InBody 720) who were drawn as a random sample. Systolic and diastolic blood pressures in the right arm are measured three times using validated electronic sphygmomanometer and a standard epidemiological method. The child is seated comfortably for at least $5 \mathrm{~min}$ prior to the first reading with $1 \mathrm{~min}$ between each measurement. Systolic blood pressure and diastolic blood pressure were recorded.

\section{Phase II}

\section{PA and SB (accelerometer)}

The ActiGraph GT3X accelerometer (ActiGraph, Pensacola, Florida, USA) is used to determine the cumulative time spent each day in activity at all intensity levels. Participants are instructed to wear the accelerometers all times during waking hours for seven consecutive days, except during water activities (such as swimming and showering) or sleeping. The accelerometers are attached to an elastic belt and worn above the iliac crest on the right side. Meanwhile, participants are asked to fill in a PA log-provided detailed information of PA and SB corresponding to the accelerometer records. Accelerometer data are analysed by software (ActiLife V.6.13.3). The following cut-off points ${ }^{22}$ for metablic equivalent (METs) were used to define light-intensity, moderate-intensity and vigorous-intensity PA using the Actigraph accelerometer: sedentary: $<1.5$ MET $(0-149$ counts/min); light: $1.5 \leq$ MET $<3$ (150-499 counts/min); moderate: $3 \leq$ MET $<6$ (500-3999 counts/min); vigorous: $\leq 6$ MET (400 or more counts/min). Total wear time was recorded and averaged per day. Data are analysed for participants who met the minimum wear time criteria ( $\geq 10$ hours on $\geq 3$ days).

\section{Cognitive tasks}

\section{Corsi block-tapping task}

Corsi block-tapping task is widely used as a representative of visuospatial attention and working memory. A traditional Corsi board structure shows nine yellow square frames on a black background. In the forward condition, participants tap the squares in the same serial order, from the first block that flashed to the last one. In case of a backward procedure, they are required to tap the blocks in a reverse serial order. Starting from sequences of two items, if at least one of these is repeated correctly, the next two trials of a sequence of an increased length will be administered. Participants' span is defined by the last sequence length that the participant repeats with one or no errors before the task is terminated. Three accuracy measures are registered: the number of blocks of the longest correct sequence, the total number of correctly reproduced sequences and the product of these two measures.

\section{Stroop task}

The Stroop task is particularly useful for investigating attention control. We use three components with five colours in the experiment (red, green, blue, grey and purple). In the first part, the black word is the name of colour shown on the computer screen, and participants have to read out the meaning of the word. Next, the pattern@@@@ served as a non-letter neutral stimulus, and participants have to identify the colour of ink. In the last part, participants are asked to mentally identify the same colour that is shown and to ignore the printed written words on the screen. Participants are instructed to respond as quickly and as accurately as possibly via the keyboard (buttons are marked with coloured stickers). Response accuracy and reaction time are collected to assess behavioural performance.

\section{Wisconsin Card Sorting Test}

The Wisconsin Card Sorting Test is a commonly used measure of planning, organisation, cognitive shifting, goal-oriented behaviour and inhibition. The test consists of four stimulus cards, each with a different colour (red, green, blue and yellow), form (circle, triangle, cross and star) and number of shapes (one, two, three and four). Participants are administered 128 cards with various colours, forms and numbers of shapes on them and are required to match each of those cards with a stimulus card, according to which they consider correct. They are informed whether or not they are correct after each sort. The first sorting category is number, and after 10 consecutive correct sorts, the category changes to form, without forewarning, and then accordingly, to colour. There are several potential scoring measures, including categories completed, perseverative errors, non-perseverative errors and failure to maintain set.

\section{Cardiorespiratory fitness}

Fitness testing is undertaken on the participants using the multiphase $20 \mathrm{~m}$ shuttle run. We record the total laps and maximum run speed (MRS) completed as an indicator of his or her cardiorespiratory fitness. Estimations of maximal oxygen consumption $\left(\mathrm{VO}_{2 \max }\right)$ were obtained using Léger's equation $\left(\mathrm{VO}_{2 \max }=31.025+3.238 \times\right.$ MRS-3.248 $\times$ age $+0.1536 \times$ MRS $\times$ age $).{ }^{23}$

\section{Combined Raven's Test}

The intelligence of children is initially assessed using the Combined Raven's Test (second edition). Each participant is given a booklet and a sheet of answer paper in quiet classrooms by group testing. Participants are guided by trained research assistants and have $40 \mathrm{~min}$ to finish this test. 


\section{Phase III}

Wechsler Intelligence Scale for Children-Fourth Edition

The WISC-IV is a psychometric measure of intelligence for children aged 6 to 16 years old, including four subscales of verbal comprehension, perceptual reasoning, working memory and processing speed. A validity study of the revision of WISC-IV Chinese version has been conducted by Zhang Houcan in $2009 .^{24}$

\section{Event-related potentials}

ERPs is a neuroimaging technique examining electroencephalographic activity used to identify structural and functional mechanisms, ${ }^{25}$ and Go/Nogo paradigm have been used in previous studies indicating that the test can detect changes in inhibition and attentional control. ${ }^{26}$ ERPs will be recorded trough EEG during a Go/Nogo paradigm. Participants are instructed to respond by pressing a button contingent on the visual presentation of the letter ' $\mathrm{M}$ ' on a computer screen, which appeared in $80 \%$ of the trials (Go condition). Conversely, participants are instructed to withhold the response when the letter ' $\mathrm{W}$ ' is presented (Nogo condition, 20\% of the trials). Overall 400 Go and Nogo cues are presented. A large percentage of false alarms, that is, pressing the response button upon presentation of the letter ' $\mathrm{W}$ ', is presumed to indicate a reduced behavioural performance of inhibitory control. EEG is recorded with a sampling rate of $1000 \mathrm{~Hz}$ from 30 silver/silver chloride electrodes (FP1, FP2, F7, F3, FZ, F4, F8, FT7, FC3, FCZ, FC4, FT8, T7, C3, CZ, C4, T8, TP7, CP3, CPZ, CP4, TP8, P7, P3, PZ, P4, P8, O1, OZ, O2) using Brainamp amplifier (Brain Products, Gilching, Germany). In addition, vertical and horizontal electro-ocular activity are recorded by two additional pairs of electrodes. The software package VisionAnalyzer V.2.04 is used to analyse the data.

\section{Follow-up and retention strategy}

We will investigate same participants from the five primary schools at biennial follow-up. We plan to follow-up all participants until they graduate from high school. All three phases are performed in follow-up assessments and the process are identical to the baseline. Data collected from the cohort are summarised in table 2 .

To reduce the loss of follow-up, some incentives are provided. All schools completing the trial will be honoured as 'model schools' by School of Public Health, Sun Yat-sen University. The class teachers and school doctors involved receive some financial charges or gifts. All the students who participated in the blood sample collection have a free breakfast and some presents. In particular, we provide feedback to the participants and their parents about the results of all measurements.

\section{Quality control}

To evaluate the effectiveness of epidemiological methods and to find out possible problems, a pilot study was conducted among 298 students randomly selected from first to sixth grade for a preliminary investigation in April 2017. Before the commencement of full-scale study recruitment, all research assistants have been trained on the investigation purpose, plan, process, relevant knowledge and data collection skills. Then school doctors and head teachers attended a face-to-face appointment to discuss the study and trained by the professional members of the research team. On the other hand, appropriate help and guidance are given to parents through WeChat as effectively as possible. Data are recorded according to standardised methods and using calibrated instruments. About 5\% students are rechecked randomly, it should be corrected if any problem is found, and all the students have to be measured again if the error exceeds $10 \%$.

Table 2 Summary of the outcomes

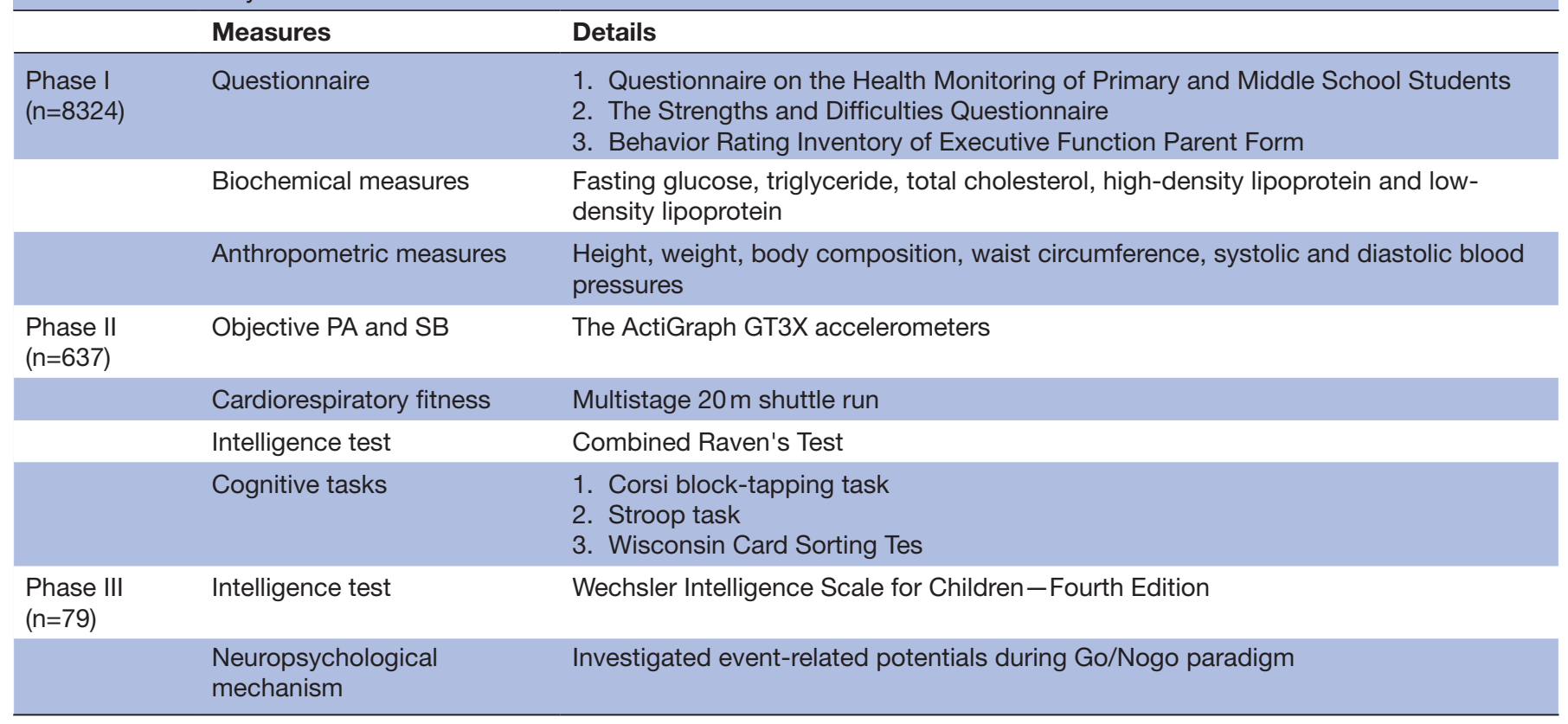




\section{Ethics and dissemination}

All parents or guardians of participants signed the informed consent form voluntarily before taking part in the study. Participants' privacy is protected throughout the study process. The findings of the study will be published in peer-reviewed journals.

\section{Patient and public involvement}

We did not involve patients or the public in our work.

\section{Statistical analyses}

Questionnaires are entered twice to ensure the accuracy of the data recorded with EpiData V.3.1. Analyses are performed with statistical software SAS V.9.4 or SPSS V.21.0. To determine potential differences between those who attend the re-examinations and those who have been lost to follow-up, unplanned missing data are evaluated. Cross-sectional analyses at both baseline and follow-up are conducted. Differences between groups are determined by analysis of variance or $\chi^{2}$ test. We plan to employ multiple linear regression models and multiple logistic regression models to examine the association of PA and SB with health-related variables, such as cognition, and cardiometabolic risk factors. Analyses also will involve isotemporal substitution models that examined the association of substituting $30 \mathrm{~min}$ (or some other length of time) each intensity category with an equal amount of time spent in the other intensity category. Further, the cohort study design allows various hypothesis to be tested longitudinally. Change analyses are considered whether changes in $\mathrm{PA} / \mathrm{SB}$ are related to changes in outcomes.

\section{DISCUSSION}

Prevalence of sufficient PA among students in China from 2005 to 2018 was estimated as $31.1 \% .{ }^{27}$ The average time of SB was 2.92 hours/day from the data of 2010-2012 China National Nutrition and Health Surveillance. ${ }^{28}$ Moreover, study found increasing trend in SB among Chinese schoolchildren. ${ }^{29}$ It makes great sense to study PA and SB because China is suffering the burden caused by the transition of lifestyle and behaviour patterns among schoolaged children. ${ }^{30} \mathrm{SB}$ is defined as any waking behaviour characterised by an energy expenditure $\leq 1.5$ metabolic equivalents while in a sitting or reclining posture. ${ }^{31}$ Previous study showed that the potential role of SB in physical and mental health was different from either low levels PA or lack of PA. ${ }^{32}$ The independent and combined associations between $\mathrm{PA}$ and $\mathrm{SB}$ with health outcomes are far less clear. Is it right to emphasise increasing PA while ignoring SB or merely reducing SB? Therefore, we designed to stratify our samples into four subgroups with different $\mathrm{SB}$ and PA levels so that PA and SB can be studied independently, and combined associations also can be studied by coexposures. The best advice for children is what we are looking for.

Emerging evidence suggested that all intensities of PA (including light-intensity PA) were important and different for health promotion and disease prevention. ${ }^{33}$ Meanwhile, different types of SB have different associations with cognitive function. ${ }^{35}$ Previous studies paid more attention to the associations between MVPA or SB volume and cognitive performance, ${ }^{32}{ }^{33}$ but the evidences of different forms or patterns are limited. In our study, PA and SB are measured using triaxial accelerometer for accurate and objective information of intensities and duration. In addition, we designed a PA-log to provide qualitative detail about the forms or patterns of $\mathrm{PA}$ and SB. What are the best varieties, intensities, frequencies and durations of PA and SB? Our research might be able to offer some suggestions.

The longitudinal study also will track cardiometabolic risk factors that provide strong evidence to determine the relationship between PA and SB on cardiometabolic risk factors. On the other hand, the study will explore the relationship between cardiometabolic risk factors and poor cognitive function. Progression of neurocognitive impairment is associated with increasingly severe manifestations of cardiovascular risk and disease in older adults. ${ }^{36}$ Cardiovascular risk and disease have been less investigated as a potential predictor of cognitive health in children. Although cardiovascular diseases typically do not emerge before adulthood, an earlier sign of cardiovascular diseases, cardiometabolic risk factors, appeared to be related with cognitive function and can be reversed during the early life course. ${ }^{37}$ Improved cardiometablic risk profile may be one explanation how $\mathrm{PA}$ and $\mathrm{SB}$ affects on cognition and brain health.

What have to mention is that, since there were less studies addressed the potential neurophysiological features and mechanisms in children, ${ }^{38}$ we planned to explore the brain levels mechanisms by investigating changes in ERPs which will be recorded using EEG during Go/Nogo paradigm. Neurophysiological studies have revealed differences in cognitive function that are related to PA behaviour. Examination of EEG has revealed that PA increased activation or higher mean frequency in spectral bands. P3 as a component of the ERPs, generated by a network of neural structures, is especially sensitive to changes in PA participation and aerobic fitness. ${ }^{13}$ Thus, using EEG to reflect changes in the brain can explore how PA and SB influence cognitive function.

Several strengths of this study are as follows. First, this study is a prospective design providing greater power to determine the varying effects of PA and SB. Second, we take into account normal children in various development phases which enable the researchers to investigate the changes in physical and neurobehavioural development among the distinct subgroups of exposed children. Then, we use a combination of subjective and objective measurements to provide quantitative and qualitative details from multiple dimensions.

An apparent weakness of this consortium is loss to follow-up. School-based setting is a valid option, which includes entire groups of children and adolescents, and provides an ideal and easily accessible environment which 
can effectively reduce the loss of visits. The health Promotion Center for Primary and Secondary Schools of Guangzhou Municipality, a subordinate body of Education Bureau, has registered the information of all students in Guangzhou. The registers can be used to follow the participants and to evaluate potential differences between those who attend the re-examinations and those who have been lost to follow-up. Second, weather and climate may have an influence on PA. We conduct the questionnaire survey every time from March to May when the weather and climate conditions are relatively stable in Guangzhou to control these factors. Third, social development in Guangzhou is at a more advanced stage compared with some parts of China. Generalising findings from our study to the nation may be limited.

It is apparent that the available data can provide a greater understanding of the role of $\mathrm{PA}$ and $\mathrm{SB}$ in determining the development of cardiometabolic risk factors and cognitive function. The findings of this study may lead to more scientific and effective early treatment and primary prevention strategies on PA and SB in schools.

Acknowledgements We would like to acknowledge the kindly support from all participated students, parents, local education, health staff and team members.

Contributors $\mathrm{YL}$ is the first author. $\mathrm{LC}$ and $\mathrm{YC}$ obtained funding. LC, ZG, XZ and YL designed the study. YL, LC, ZG, XZ, MT, NW, LL, SL and WT collected the data. $Y L$ drafted the manuscript. $L C$ and $Y C$ contributed to the interpretation of the results and critical revision of the manuscript for important intellectual content and approved the final version of the manuscript. All authors have read and approved the final manuscript. $Y C$ is the study guarantor.

Funding This study is supported by the National Natural Science Foundation of China (Grant No. 81673193).

Competing interests None declared.

Patient consent for publication Not required.

Ethics approval Procedures described in this manuscript have been approved by the Ethical Review Committee for Biomedical Research, School of Public Health, Sun Yat-sen University (L2016-010).

Provenance and peer review Not commissioned; externally peer reviewed.

Open access This is an open access article distributed in accordance with the Creative Commons Attribution Non Commercial (CC BY-NC 4.0) license, which permits others to distribute, remix, adapt, build upon this work non-commercially, and license their derivative works on different terms, provided the original work is properly cited, appropriate credit is given, any changes made indicated, and the use is non-commercial. See: http://creativecommons.org/licenses/by-nc/4.0/.

ORCID iD

Yajie Lv http://orcid.org/0000-0001-9598-920X

\section{REFERENCES}

1 Pujadas Botey A, Bayrampour H, Carson V, et al. Adherence to Canadian physical activity and sedentary behaviour guidelines among children 2 to 13 years of age. Prev Med Rep 2016;3:14-20.

2 World Health Organization. Levels of insufficient physical activity, 2018. Available: http://www.who.int/news-room/fact-sheets/detail/ physical-activity [Accessed 22 Feb 2019].

3 Hallal PC, Andersen LB, Bull FC, et al. Global physical activity levels: surveillance progress, pitfalls, and prospects. The Lancet 2012;380:247-57.

4 Andersen LB, Riddoch C, Kriemler S, et al. Physical activity and cardiovascular risk factors in children. $\mathrm{Br} J$ Sports Med 2011;45:871-6.

5 Ekelund U, Luan Jian'an, Sherar LB, et al. Moderate to vigorous physical activity and sedentary time and cardiometabolic risk factors in children and adolescents. JAMA 2012;307:704-12.
6 Saunders TJ, Chaput J-P, Tremblay MS. Sedentary behaviour as an emerging risk factor for cardiometabolic diseases in children and youth. Can J Diabetes 2014;38:53-61.

7 de Greeff JW, Bosker RJ, Oosterlaan J, et al. Effects of physical activity on executive functions, attention and academic performance in preadolescent children: a meta-analysis. J Sci Med Sport 2018;21:501-7.

8 Donnelly JE, Hillman $\mathrm{CH}$, Castelli D, et al. Physical activity, fitness, cognitive function, and academic achievement in children: a systematic review. Med Sci Sports Exerc 2016;48:1197-222.

9 Diamond AB. The cognitive benefits of exercise in youth. Curr Sports Med Rep 2015:14:320-6.

10 Falck RS, Davis JC, Liu-Ambrose T. What is the association between sedentary behaviour and cognitive function? A systematic review. $\mathrm{Br}$ J Sports Med 2017;51:800-11.

11 Mehta R, Shortz A, Benden M. Standing up for learning: a pilot investigation on the neurocognitive benefits of Stand-Biased school Desks. Int J Environ Res Public Health 2016;13:59.

12 Carson V, Kuzik N, Hunter S, et al. Systematic review of sedentary behavior and cognitive development in early childhood. Prev Med 2015;78:115-22.

13 Hillman $\mathrm{CH}$, Erickson $\mathrm{Kl}$, Kramer AF. Be smart, exercise your heart: exercise effects on brain and cognition. Nat Rev Neurosci 2008:9:58-65.

14 Liang J, Matheson BE, Kaye WH, et al. Neurocognitive correlates of obesity and obesity-related behaviors in children and adolescents. Int J Obes 2014;38:494-506.

15 Corley J, Starr JM, Deary IJ. Serum cholesterol and cognitive functions: the Lothian birth cohort 1936. Int Psychogeriatr 2015;27:439-53.

16 Rofey DL, Arslanian SA, El Nokali NE, et al. Brain volume and white matter in youth with type 2 diabetes compared to obese and normal weight, non-diabetic Peers: a pilot study. Int J Dev Neurosci 2015;46:88-91.

17 Lande MB, Kupferman JC. Cognitive function in hypertensive children. Curr Hypertens Rep 2015;17:508.

18 Cannon JA, Moffitt P, Perez-Moreno AC, et al. Cognitive impairment and heart failure: systematic review and meta-analysis. J Card Fail 2017;23:464-75.

19 Kerola T, Kettunen R, Nieminen T. The complex interplay of cardiovascular system and cognition: how to predict dementia in the elderly? Int J Cardiol 2011;150:123-9.

20 Kou J, Du Y, Xia L. Reliability and validity of "children strengths and difficulties questionnaire" in Shanghai norm. Shanghai Spirit Medicine 2005;01:25-8

$21 \mathrm{Li} \mathrm{Y-P,} \mathrm{Hu} \mathrm{X-Q,} \mathrm{Jing} \mathrm{Zhao,} \mathrm{et} \mathrm{al.} \mathrm{Application} \mathrm{of} \mathrm{the} \mathrm{who} \mathrm{growth}$ reference (2007) to assess the nutritional status of children in China. Biomed Environ Sci 2009;22:130-5.

22 Freedson P, Pober D, Janz KF, et al. Calibration of accelerometer output for children. Med Sci Sports Exerc 2005;37:S523-30.

23 Léger LA, Mercier D, Gadoury C, et al. The multistage 20 metre shuttle run test for aerobic fitness. J Sports Sci 1988;6:93-101.

24 Zhang H. The revision of WISC-IV Chinese version. Psychological Science 2009;32:1177-9.

25 Chaddock L, Pontifex MB, Hillman $\mathrm{CH}$, et al. A review of the relation of aerobic fitness and physical activity to brain structure and function in children. $J$ Int Neuropsychol Soc 2011;17:975-85.

26 Rosburg T, Deuring G, Boillat C, et al. Inhibition and attentional control in pedophilic child sexual offenders - An event-related potential study. Clin Neurophysiol 2018;129:1990-8.

27 Wang N, He J, Wang Z, et al. The prevalence of sufficient physical activity among primary and high school students in mainland China: a systematic review and meta-analysis. Public Health 2018:163:67-75

28 Song C, Guo H, Gong W, et al. [Status of sedentary activities in the leisure time in Chinese pupils in 2010-2012]. Wei Sheng Yan Jiu 2017;46:705-21.

29 Dearth-Wesley T, Howard AG, Wang H, et al. Trends in domainspecific physical activity and sedentary behaviors among Chinese school children, 2004-2011. Int J Behav Nutr Phys Act 2017;14.

30 Wang L, Wang L. Using theory of planned behavior to predict the physical activity of children: probing gender differences. Biomed Res Int 2015;2015:1-9.

31 Sedentary Behaviour Research Network. Letter to the editor: standardized use of the terms "sedentary" and "sedentary behaviours". Appl Physiol Nutr Metab 2012;37:540-2.

32 Elmesmari R, Reilly JJ, Martin A, et al. Accelerometer measured levels of moderate-to-vigorous intensity physical activity and sedentary time in children and adolescents with chronic disease: a systematic review and meta-analysis. PLoS One 2017;12:e179429. 
33 Poitras VJ, Gray CE, Borghese MM, et al. Systematic review of the relationships between objectively measured physical activity and health indicators in school-aged children and youth. Appl Physiol Nutr Metab 2016:41:S197-239.

34 Álvarez-Bueno C, Pesce C, Cavero-Redondo I, et al. The Effect of Physical Activity Interventions on Children's Cognition and Metacognition: A Systematic Review and Meta-Analysis. J Am Acad Child Adolesc Psychiatry 2017;56:729-38.

35 Aggio D, Smith L, Fisher A, et al. Context-Specific associations of physical activity and sedentary behavior with cognition in children. Am J Epidemiol 2016;183:1075-82.
36 Waldstein SR, Wendell CR. Neurocognitive function and cardiovascular disease. J Alzheimers Dis 2010;20:833-42.

37 Wideman L, Calkins SD, Janssen JA, et al. Rationale, design and methods for the right track health study: pathways from childhood self-regulation to cardiovascular risk in adolescence. BMC Public Health 2016;16:459.

38 Lubans D, Richards J, Hillman C, et al. Physical activity for cognitive and mental health in youth: a systematic review of mechanisms. Pediatrics 2016;138:e20161642. 